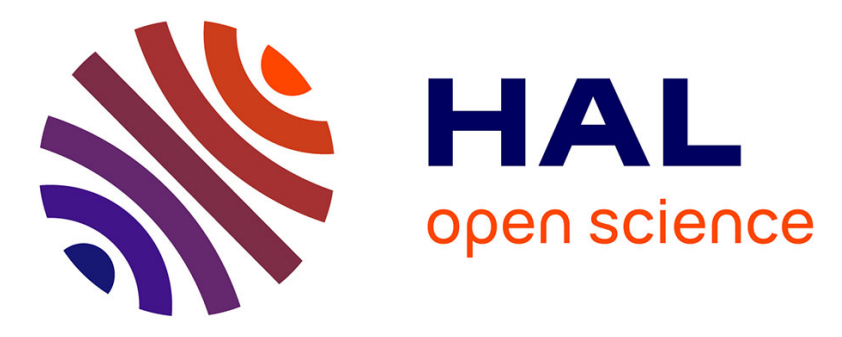

\title{
Système métropolitain et logique de projet : une approche de la question urbaine en géographie et aménagement
}

\author{
Anne-Peggy Hellequin, Nathalie Lemarchand, Didier Paris
}

\section{To cite this version:}

Anne-Peggy Hellequin, Nathalie Lemarchand, Didier Paris. Système métropolitain et logique de projet : une approche de la question urbaine en géographie et aménagement. Territoire en mouvement. Revue de Géographie et d'Aménagement, 2006, 1, pp.34-44. 10.4000/tem.120 . halshs-02939869

\section{HAL Id: halshs-02939869 \\ https://shs.hal.science/halshs-02939869}

Submitted on 15 Sep 2020

HAL is a multi-disciplinary open access archive for the deposit and dissemination of scientific research documents, whether they are published or not. The documents may come from teaching and research institutions in France or abroad, or from public or private research centers.
L'archive ouverte pluridisciplinaire HAL, est destinée au dépôt et à la diffusion de documents scientifiques de niveau recherche, publiés ou non, émanant des établissements d'enseignement et de recherche français ou étrangers, des laboratoires publics ou privés. 


\section{OpenEdition}

Journals

Territoire en mouvement

Revue de géographie et aménagement

Territory in movement Journal of geography and planning

1 | 2006:

Perspectives

Articles

\section{Système métropolitain et logique de projet : une approche de la question urbaine en géographie et aménagement}

Metropolization and Urban Project Rationality: a Geographical and Urban Planning Approach

Anne-Peggy Hellequin, Nathalie Lemarchand et Didier Paris

p. $34-44$

https://accesdistant.bu.univ-paris8.fr:2118/10.4000/tem.120

\section{Résumés}

Français English

Dans cet article nous avons cherché à préciser les contours du champ de recherche que constitue la ville afin d'en dégager des pistes de réflexion pour la revue Territoire en Mouvement. Une première observation montre que la réflexion scientifique sur la question urbaine a souvent accompagné la production des discours politiques. Sur le plan spatial, l'on remarque que les territoires métropolitains sont présentés comme des systèmes complexes où se juxtaposent des polarités économiques et sociales. La métropolisation constituerait ainsi un modèle de développement «territorialisé » dans lequel agissent en synergie les acteurs du développement local. Quant il prend forme, le projet urbain intervient alors comme un paradigme qui porte le dessein d'une ville, la forme de ses espaces mais aussi un projet de société. Les expérimentations menées dans les villes du Nord - Pas de Calais, et au sein du vaste système métropolitain de la région urbaine de Lille, couvrent l'ensemble des termes de cette réflexion; ils ont aussi valeur de modèle à l'exemple de la notion de renouvellement urbain expérimentée dans cette région.

This article can be seen as an attempt to identify the contours of urban studies in such a way that the journal Territoire en Mouvement could precise its own working path. A first general observation shows that the scientific thought on the urban phenomenon has often gone along 
with the political discourses. In a spatial perspective, urban territories are often described as complex systems where economic and social components overlap. In this way, the metropolization process can be seen as a territorialized developmental model, in which local actors are forced to act in synergy. Where it emerges, the urban project thus becomes a paradigm for the discussion on its design, its places and on the social project it bears. Experimentations in the Nord - Pas de Calais region north of France, most notably within the metropolitan region of Lille, provides god insights in this debate, as shown by the fact that the notion of urban renewal was first put forward in the region.

\section{Entrées d'index}

Mots-clés : ville, métropolisation, Lille, étude urbaine, Nord - Pas de Calais

Keywords : metropolization, Lille, urban study, Nord - Pas de Calais

\section{Texte intégral}

\section{Introduction}

Parce que les territoires urbains sont, en Europe, le lieu de vie de la majorité de la population, ils font l'objet d'une observation attentive des équipes de recherches en géographie et aménagement. Dans un contexte régional de l'Europe du nord-ouest caractérisé par de fortes densités urbaines et le déploiement de systèmes urbains complexes, les chercheurs du Nord de la France se sont saisis de longue date de cet objet, apportant ainsi leur contribution à une production scientifique française très abondante sur le sujet. Le propos de cet article est de préciser les cadres de la recherche dans le champ de l'urbain qui pourront, pour l'avenir, tracer des pistes de réflexion pour la revue Territoires en Mouvement. Grâce à l'existence de lieux d'échanges scientifiques, tel l'IFRESI-CNRS à Lille, cette production scientifique, qui associe également sociologues, anthropologues, économistes, politistes, architectes, historiens.... est fondamentalement marquée par son caractère pluridisciplinaire.

Comme l'a montré M. Lussault, (2000), la géographie urbaine a été profondément renouvelée à la fin des années soixante à la faveur notamment de l'article de P. Claval de 1968, repris dans son ouvrage de 1982 et des apports de la sociologie et de l'économie. À partir de ce tournant, M. Lussault distingue deux postures scientifiques " interniste » et " externiste » de compréhensions des espaces urbanisés. Cette distinction nous servira de ligne de partage entre les différents travaux qui sont menés dans les universités du Nord - Pas de Calais. On peut ainsi distinguer un premier champ qui repose sur des études " externistes » qui s'intéresse à la métropolisation, donc à la compréhension de la ville dans un espace plus large, et d'autre part un second champ qui s'intéresse lui plutôt aux formes urbaines c'est-à-dire aux espaces de la métropolisation et aux représentations spatiales des acteurs prises à travers leurs discours.

\section{L'irruption de la question métropolitaine sur les scènes politique et scientifique}

C'est au cours des années 1980 que la problématique de la métropolisation s'est imposée dans les pratiques politiques destinées à accompagner la mutation des villes. En France, le renforcement des compétences locales, dans le cadre de la décentralisation, avait modifié la relation au pouvoir central et multiplié les acteurs locaux du développement des territoires. Par ailleurs, les perspectives ouvertes par le processus de construction européenne faisaient émerger un discours relatif au renforcement de la concurrence entre les territoires, en particulier entre les grandes villes d'Europe. De Georges Frêche à Montpellier (Antigone), à Pierre Mauroy à Lille 
(Euralille), un certain nombre d'élus locaux commençaient à percevoir les enjeux de ce modèle de développement métropolitain pour leur ville. Au même moment à Lyon, la révision du schéma directeur renouvelle le genre en conférant au document une vision prospective pour le développement de la métropole.

Sur le plan scientifique, la constitution d'un corpus de réflexion sur la question métropolitaine, sur lequel nous ne pouvons nous étendre de façon exhaustive ici, a souvent accompagné la production du discours politique. Animée par R. Brunet, la « Maison de la Géographie » à Montpellier, reconnue avant tout pour la qualité de son travail scientifique, a aussi participé au débat local en même temps que national, répondant notamment aux interrogations de la DATAR sur le positionnement des villes françaises en Europe (Brunet 1989), travaux réactualisés en 2003 (Rozenblat, Cicille, 2003). À Lille, les travaux de J.F. Stevens pour le compte du SGAR Nord - Pas de Calais (Stevens 1989), et ceux de C. Gachelin pour l'Agence de Développement et d'Urbanisme de Lille-Métropole, ont apporté les éléments de cadrage théorique nécessaires à la mise en oeuvre d'une stratégie de développement métropolitain. En 2006, les travaux de l'APERAU sous la direction de A. Motte, orientés sur la question de la mise en œuvre des projets de coopération métropolitaine s'articulent également aux questionnements de la DATAR, devenue DIACT (Motte et alii, 2006).

Enfin, à partir de la fin des années 1980 et du début des années 1990 jusqu’à aujourd'hui, des travaux théoriques parmi lesquels ceux de Pierre Veltz (1997), François Asher (1995), Bernard Jouve et Christian Lefebvre (2002), Alain Bourdin, ont permis de mieux comprendre les dynamiques métropolitaines dans le contexte contemporain de transformation urbaine. Par ailleurs, au sein de collections dédiées aux métropoles, de Marseille (Morel, 1999) à Lille (Bruyelle, 1991 ; Paris \& Stevens, 2000), de Bordeaux (Godier \& Tapie, 2004) à Toulouse (Jalabert, 1995) et autres, des travaux plus empiriques permettaient d'analyser localement les processus de développement métropolitain, alimentant un corpus de connaissance à partir des principaux terrains d'étude.

$6 \quad$ Le fait métropolitain constitue un élément central dans la compréhension des mécanismes contemporains de développement territorial. Lieu d'articulation entre le " global » et le « local/régional », la métropole concentre les ressources nécessaires à l'alimentation du processus " post-fordiste » de développement des territoires, ce qui n'enlève rien à la capacité du fordisme à se renouveler à travers des formes néofordistes (exemple des « call center ») qui coexistent avec les formes nouvelles de développement fondées sur d'autres bases (ex : la culture, la créativité...). Par ailleurs, la métropolisation représente le cadre conceptuel auquel se réfèrent nombre d'acteurs du développement. Le concept renvoie aussi bien aux modalités et processus du développement métropolitain qu'à la problématique de la diffusion et de l'organisation spatiale (Paris, 2003).

\section{La complexité du système métropolitain}

Sur le plan spatial, les territoires métropolitains apparaissent comme des systèmes complexes où se juxtaposent des polarités économiques et sociales variées. Les métropoles sont des lieux privilégiés de production de richesses, et celles-ci se répartissent de façon inégale selon une logique de différenciation spatiale, ce qui n'est pas sans conséquences pour la forme urbaine elle-même. Les pôles économiques « métropolitains » ou « technopolitains » concentrent les fonctions tertiaires de haut niveau, les activités de recherche- développement, de la finance dans des centres d'affaires ou des parcs d'activités spécialisées qui inscrivent le projet métropolitain dans le paysage urbain, à l'exemple d'Euralille. En négatif, des espaces délaissés par l'ancienne industrie constituent dans le meilleur des cas des opportunités foncières pour de nouveaux développements et dans le pire des cas des friches dont le coût de remise en état ou la localisation représentent un handicap dans la perspective d'un nouvel usage. Les contrastes entre les inclus et les exclus du développement métropolitain sont le fondement de la géographie sociale de ces territoires. Quartiers 
chics ou destinés à la classe moyenne s'opposent aux quartiers de relégation selon le processus largement orienté par le marché immobilier. Ce dispositif engendre la multiplication des discontinuités au sein du territoire métropolitain.

À une autre échelle, l'espace métropolitain s'inscrit dans un cadre régional où la grille de lecture traditionnelle opposant centre et périphérie, qui peut encore être convoquée pour certains cas (Toulouse) laisse le plus souvent la place à un système d'interaction complexe entre différents pôles urbains intégrés au sein de régions urbaines de forte densité (région lilloise, lyonnaise...) : Par son ampleur, le cas de Paris et de l'Île-deFrance apparaît spécifique dans le cadre hexagonal. C'est dans ce contexte que la DIACT a lancé un appel à coopération métropolitaine destinée à mieux organiser ces vastes ensembles autour d'initiatives à caractère structurant (Motte et alii, 2006), à l'exemple de la Région Urbaine de Lyon (RUL), de l'aire métropolitaine de Lille, ou du projet "Côte d'Opale-Flandre occidentale Belge », complémentaire ou qui se veut concurrente de celui de l'aire métropolitaine de Lille au sein d'une grande région urbaine transfrontalière (Hellequin, Duhamel, 2005).

$9 \quad$ Parmi les éléments participant à l'établissement de ces systèmes complexes en produisant de la polarité économique et sociale dans la métropole, on peut prendre l'exemple du centre commercial. En l'espace de 40 ans, les centres commerciaux ont progressivement occupé la périphérie et établi un nouvel équipement familier dans le paysage de nos banlieues. Il constitue ainsi un des trois éléments structurants de la périphérie ; centre commercial, zone d'activités et lotissements pavillonnaires. Couvrant toujours plus largement les besoins des populations métropolitaines, ils complètent l'offre commerciale avec des grandes et moyennes spécialisées, au point de devenir des «parcs commerciaux ». Ils accompagnent l'étalement urbain et couvrent un bassin de chalandise s'étendant de plus en plus loin, à l'échelle de la région pour les plus grands. Malgré les tentatives de l'État ou des villes centres, les périphéries métropolitaines se sont dotées peu à peu de ces équipements au rythme du développement de la société de consommation. Ceux-ci ont aujourd'hui une surface commerciale supérieure à celle dont dispose la ville centre. Benoît Latreille $(\mathrm{C} \& \mathrm{~W} / \mathrm{H} \& \mathrm{~B})$ le dit très bien : "Sur les 65 millions de mètres carrés qu'occupe le commerce, les parcs d'activités représentent à eux seuls 36 millions. Et les ménages y réalisent $30 \%$ de leurs dépenses. ${ }^{1}$

Aujourd'hui l'équipement commercial métropolitain se diversifie : centre commercial et de loisirs ou centre commercial thématique s'installent à la périphérie des métropoles disposant d'un bassin de consommateurs très élevé en densité et en pouvoir d'achat, mais aussi d'une situation géographique lui amenant une clientèle touristique supplémentaire. Si la première génération française de centre commercial associant commerces et loisirs comporte seulement des cinémas multiplexes en matière de loisirs, désormais ce complément au commerce devient insuffisant. En effet, la dimension ludique doit se décliner à travers des installations sportives (pistes de ski, patinoire), ludiques (bowling) ou muséographiques (planétarium, aquarium) accompagnant un pôle restauration comptant quelques dizaines de restaurants et une galerie de plus de 150 magasins. Ces équipements, inaugurés au Canada avec le West Edmonton Mall en $1983^{2}$, prennent pied en Europe (Lisbonne, Londres et Barcelone) à partir de 1999, avant de rejoindre la France en 2006. C'est à Montpellier (Odyseum 90000 m2) que la formule s'installe. C'est aujourd'hui, le seul projet périphérique. Lyon ou Marseille ont des projets similaires mais dans les quartiers proches du centre.

Si l'on considère la métropole lilloise, elle compte une structure commerciale multipolaire composée de 5 grands pôles commerciaux périphériques et du quartier commercial de centre ville. Ces pôles renforcent l'attractivité de la métropole sur la région ; les agglomérations de Valenciennes, de Béthune, d'Arras et même de Dunkerque connaissent une évasion certaine de leur clientèle au profit de la métropole. Gérard Mulliez calcule que "Lille est au cœur d'une zone de chalandise d'environ 3 millions de consommateurs vivant de part et d'autre de la frontière belge. » Il ajoute que : « le passage des TGV nord-européens qui complètent un réseau autoroutier très dense, permet à Lille d'exploiter pleinement cette situation géographique exceptionnelle. » Sans oublier qu' " historiquement, les échanges transfrontaliers ont toujours fait partie de la vie quotidienne des habitants de ce territoire : quand les 
salaires étaient plus élevés en France, les Belges venaient travailler dans la métropole lilloise et vice-versa. »3

12 La rénovation du quartier historique de la métropole régionale et l'extension $\mathrm{du}$ centre-ville avec Euralille, centre d'affaires et commercial connecté à la gare TGV, y a dynamisé le commerce. Le secteur piétonnier accueille chaque samedi plus de 80 ooo personnes. Les transactions commerciales attestent qu'il est devenu l'un des plus chers de province4. Cette transformation s'est accompagnée d'un regroupement et d'une spécialisation des enseignes par quartiers et par niveau de gammes qui a certes renforcé l'attraction du centre-ville mais a entraîné parallèlement une fragmentation de ce pôle commercial central. À la périphérie, le nouvel équipement en projet est un centre commercial thématique consacré à la maison. Le premier du genre vient d'être inauguré dans la région parisienne. 5

Cette situation commerciale sur la métropole lilloise tend à démontrer " la métropolisation de l'offre commerciale » qu'évoquait J.-P. Bondue (1997) ${ }^{6}$. Celle-ci n'a pas cependant fait disparaître toutes tentatives des agglomérations régionales à reconquérir leur clientèle comprise dans leur bassin de chalandise. Les agglomérations de Valenciennes (2006) comme celle de Dunkerque (2006) s'appuient aujourd'hui sur des schémas d'aménagement commercial dans lesquels commerce de centre-ville et commerce de périphérie se soutiennent pour renforcer l'attractivité globale de l'agglomération. Elles renforcent ainsi la multipolarisation commerciale de leur territoire urbain par des opérations autorisant d'une part grandes et moyennes surfaces spécialisées en périphérie et d'autre part l'inauguration d'un centre commercial nouvelle génération dans le centre-ville, espérant par cet aménagement contrôlé de leur territoire augmenter leur capacité à conserver les dépenses de consommation de leur population.

\section{La gouvernance métropolitaine en question}

La métropolisation apparaît aussi comme un modèle de développement « territorialisé », dans lequel agissent en synergie les acteurs de développement local : acteurs politiques, monde économique, milieu culturel et universitaire, secteur associatif, etc... qui constituent la société locale. Cette approche renvoie à la notion de " gouvernance métropolitaine », qui repose sur l'idée que l'aptitude d'une société locale à faire émerger des projets conditionne le développement du territoire. Au-delà du gouvernement local, c'est-à-dire de gestion politique du territoire, la « bonne gouvernance " rend compte de la mobilisation des acteurs de ce territoire dans une perspective de projet. L'exemple de Lille dans les années 1980 et 1990 est un bon exemple qui fait référence en France et en Europe. L'enchainement Tunnel sous la Manche, TGV, projet Euralille s'est articulé à une mobilisation des acteurs de la société civile, notamment du Comité Grand Lille, et des politiques pour obtenir la gare TGV au coeur de Lille, ce qui n'était pas gagné d'avance, puis pour faire émerger une stratégie à caractère international : cela s'est concrétisé par l'épisode de la candidature olympique, acte fondateur de la mobilisation locale, puis l'obtention de la Capitale européenne de la Culture en 2004, aujourd'hui la plus grosse opération de mécénat culturel jamais réalisée en France, et sans doute en Europe.

15 Cette mobilisation, et l'importance des investissements effectués en quelques années, ont facilité la « bifurcation métropolitaine » (Paris \& Stevens, 2000) de l'ancienne agglomération industrielle. D'autres exemples en Europe illustrent ce type de processus : Barcelone, souvent citée comme modèle de référence à Lille, Bilbao, Gênes en sont d'autres excellentes illustrations.

16 Ce processus doit impliquer le politique : dans une démocratie de représentation, les élus ont la légitimité nécessaire à la décision, ce qui pose la question de la représentation politique du territoire métropolitain à la bonne échelle (Jouve \& Lefèvre, 2002). En France, la loi Chevènement tente d'apporter une réponse en termes de cohérence de gestion, mais qui reste imparfaite en terme d'expression démocratique à 
l'échelle idoine. Mais la démocratie moderne est aussi participative, ce qui rend caduque les anciens modèles fondés sur la seule autorité politique. La loi Voynet prévoit la mise en place de conseils de développement dans ces agglomérations comme nouvel outil de gouvernance, mais, à l'exemple de Strasbourg, ils ne sont pas mis en place partout.

\section{Le projet urbain, mode de production urbaine pour les métropoles}

Si le développement des métropoles repose sur des bases économiques solides, qui les rendent compétitives au sein d'une " économie d'archipel » mondialisée (Veltz, 1997), il dépend également de la capacité des acteurs locaux à définir un projet métropolitain capable de transformer structurellement les cadres culturels, sociaux, économiques et physiques dans lesquelles s'inscrit la dynamique d'évolution des villes concernées par la logique de « bifurcation métropolitaine » (Paris \& Stevens, 2000). Loin de la logique normative de la planification des années 1960-1970, le projet métropolitain se retrouve au cœur des schémas directeurs, aujourd'hui SCOT, selon une approche stratégique illustrée en France par les exemples de Lyon, puis de Lille.

Cette mutation concerne aussi bien la transformation des données concrètes (objectivables), que celle des cadres de pensée (référentiel), ce qui n'est pas sans conséquences sur les modes de faire. Ainsi, concernant la transformation physique des espaces, l'enjeu de la qualité urbaine devient une donnée essentielle dans la compétition entre villes, et particulièrement entre métropoles. Depuis le milieu des années 1980, à travers toute l'Europe, et notamment en France, la notion de projet urbain s'est imposée dans la production des discours stratégiques des villes (Rosemberg, 2000), métropoles et villes moyennes.

19 Le projet urbain apparaît comme "l'expression de la volonté politique des élus de défendre les valeurs urbaines sans nostalgie ni passéisme, en prise sur la dynamique de leur époque, le progrès et les attentes sociales » (Masboungi, 2002). On trouve à l'origine l'expérience menée à Bologne à la fin des années 1960, qui ouvre la voie à la participation des citoyens, alors qu'en France s'impose la planification technocratique avec les villes nouvelles ou la Défense. Il faut attendre les années 1970 et la question du traitement du quartier des Halles à Paris pour voir s'amorcer chez nous le débat sur la notion de projet (Ingallina, 2001).

20 L'usage de l'expression « projet » à la place de " plan » correspond à la volonté d'avoir une approche globale de l'action politique (au sens large : action qui vise à transformer) sur la ville ou le territoire. La notion intègre par ailleurs la dimension concrète de la production urbaine, de la conception à la réalisation, en passant par la concertation (entre acteurs), la participation (des habitants), la validation (politique), etc. La conduite de projet apparait d'autant plus délicate que le projet est complexe (Euralille, Euroméditerranée..).

21 Au sein d'une vaste région urbaine animée par la métropole lilloise, les villes du Nord - Pas de Calais de sont pas restées à l'écart du mouvement : si Euralille apparaît comme le projet urbain emblématique de la bifurcation métropolitaine, le centre de Roubaix s'impose comme l'un des meilleurs exemples de renouvellement urbain. Dunkerque, avec le projet " Neptune » et l'opération " Marine-Bollaert », Valenciennes avec le projet " Coeur de Ville », le nouveau tramway, où des opérations comme " les Tertiales » ou le Quartier des Canonniers, ne sont pas en reste. Calais (Espace Charost), Tourcoing (Espace Carnot), Boulogne, Lens (dans le prolongement de l'implantation du Louvres), etc... sont également traversées par la logique de projet urbain, à des degrés divers de réalisation ou de réflexion.

22 Dans le projet urbain, la cohérence territoriale, souci politique souligné par l'établissement du SCOT et autres documents d'urbanisme, implique le secteur des activités commerciales. La nature même du commerce, activité économique privée mais en relation étroite avec l'espace public le conduit à contribuer directement au 
façonnement du paysage urbain, à la périphérie et aux entrées de ville, mais aussi dans l'hypercentre dans le secteur piétonnier et/ou sauvegardé.

La transformation des espaces constitutifs de la ville ; quartier résidentiel ou quartiers économiques implique toujours d'y introduire du commerce. Le retour du credo de la mixité urbaine et sociale favorise dans tous les projets de lui réserver une place ; commerce de proximité ou commerce de centre-ville, chacun est convoqué dans le projet urbain des métropoles. Dans les quartiers résidentiels, les pouvoirs publics ont le souci de préserver ou de réinstaller un commerce de proximité participant à leurs yeux à l'établissement d'un espace social, de rencontres et d'échanges. La réhabilitation de quartiers résidentiels ou la construction de nouveaux logements, parfois dans d'anciennes friches industrielles, favorise cette politique. Un organisme consacré à la réhabilitation commerciale a été crée, EPARECA7, installé à Lille et dirigé par un architecte, il contribue à la transformation des quartiers sensibles. Au centre, les artères commerciales et les centres commerciaux doivent concourir à la qualité architecturale du centre ville, valorisant pour les unes le patrimoine urbain par leurs devantures, ou affichant pour les autres la modernité et le dynamisme métropolitain par l'établissement d'un nouveau pôle central. Ainsi Euralille regroupant commerces et bureaux, connecté aux gares lilloises est conçu pour augmenter la visibilité et la modernité du centre de la métropole.

La qualité urbanistique du commerce est un phénomène que l'on peut dater du XIXème siècle avec d'une part, le grand magasin et de l'autre le marché couvert, tous deux bénéficiant des progrès technologiques tel que le verre industriel ou la poutrelle d'acier. Ils inaugurent d'une certaine façon la valeur du lieu de vente en tant qu'objet architectural, et participent déjà à la transformation du paysage urbain, lorsque les autorités décident d'établir de nouveaux quartiers dans lesquels ils s'installent. La valeur de certains de ces bâtiments est encore attestée aujourd'hui, ils figurent dans les guides touristiques et reçoivent de nombreux visiteurs, clients ou touristes, somme toute consommateurs succédant à ces grands magasins, et sans effacer les centres commerciaux prestigieux de centre-ville, les nouveaux monuments du commerce dans les métropoles internationales sont aujourd'hui les " magasins amiraux » d'une marque. Situés sur une artère prestigieuse, ils ont vocation à afficher la valeur économique mais aussi qualitative, voire culturelle ${ }^{8}$ de la marque. Signe extérieur de son identité, le groupe commercial doit combiner la contrainte urbanistique du lieu et la visibilité marketing de sa marque, associant ainsi une fois de plus le commerce au projet urbain. Si ces magasins amiraux sont l'apanage des grandes capitales, à une échelle moindre les mégastores et les centres commerciaux sont des équipements courtisés par les élus qui voient avec eux la certitude de renforcer l'attractivité de leur centre, tout en exigeant un projet urbain de qualité.

Dans la compétition entre les villes, la qualité architecturale des lieux du commerce n'est pas le seul intérêt que représente ce secteur économique. Le commerce a aussi pour mission de favoriser la dimension ludique voire " festive » de la ville par l'animation qu'il entraîne, soit à l'occasion d'événements culturels annuels, telles que les fêtes de fin d'année ou d'événements spécifiques à la ville ; fête locale, festivals, enfin par le calendrier commercial lui-même à l'occasion des soldes. Les rues commerciales sont transformées, leur physionomie est modifiée par la construction de décors temporaires. Les devantures des magasins se doivent d'être décorées. Par exemple à l'occasion de Lille 2004 et plus récemment dans le cadre de Lille 3000 (Bombaysers de Lille), les magasins sont sollicités pour transformer leurs devantures dans l'esprit de la manifestation, certaines rues commerciales bénéficient d'un décor de rue. L'animation des vitrines à l'occasion des fêtes du calendrier prend une grande place dans la démonstration de l'événement. On augmente ainsi la visibilité du centre mais plus encore de la métropole qui compte souvent à ces occasions un plus grand nombre de visiteurs. L'intérêt que portent les acteurs du projet urbain à la scénographie commerciale dans l'animation urbaine peut être relevée par les programmes d'aide spécifique à la rénovation des façades.

26 Ces analyses des espaces urbains et plus particulièrement de la place du commerce de détail dans la ville montrent que l'enjeu des images des villes est aujourd'hui bien compris par les édiles et les acteurs économiques qui élaborent dans ce contexte des 
coalitions privé-public puissantes. Pourtant le projet urbain a aussi une dimension socio-spatiale intéressante. En effet, à travers les représentations spatiales que peuvent en avoir les acteurs, s'éclairent des nouvelles spatialités

\section{Un projet urbain au cœur des discours : des nouvelles spatialités}

Si ces réflexions portant sur la question métropolitaine se trouvent à l'interface des " saisies internes et externes » telles qu'elles ont été définies par M. Lussault (2000, 2003), une autre problématique a pu être dégagée des travaux et des axes de recherches des différents laboratoires de la région se fondant elle plus exclusivement sur une posture « interniste ». Il s'agit d'un ensemble de réflexions encore éparses mais qui ont en commun de s'intéresser aux discours et images des espaces urbains. Au sein de ces réflexions régionales, on peut distinguer ainsi un ensemble de travaux portant sur les mots de la production urbaine. Ainsi, à partir d'analyse de la maitrise d'ouvrage des projets de territoire à Dunkerque, O. Ratouis et M. Segaud ont mis en évidence l'existence de récits partagés entre les acteurs, les auteurs définissant ainsi des collectifs d'énonciation (Ratouis et Segaud, 2001). Ces derniers s'avèrent autonomes du cadre réglementaire posé par l'État dans une ville où celui-ci a été longtemps omnipotent (création portuaire et site défensif, reconstruction, sidérurgie littorale, Port Autonome)... Ces réflexions ont été poursuivies en élargissant l'analyse des représentations spatiales au travers des discours d'acteurs. Une premiere recherche fondée sur l'analyse du rapport entre " dire et faire » a souligné la diffusion et la dynamique d'un véritable vocabulaire de la réparation, S. Valcke (2003) par exemple montre ainsi comment s'est construit un champ sémantique autour de mots préfixés en « re » : requalification, renouvellement reconversion, redéploiement....

Au-delà de l'analyse du projet urbain en train de se faire et des conditions de son élaboration, les effets du projet et plus particulièrement sa réception permettent d'évoquer des nouvelles spatialités comprises comme des rapports à l'espace (Hellequin, 2006). À partir du repérage de thématiques récurrentes dans la presse locale et nationale et dans les documents de planification, on dégage des figures clés de la production urbaine pour les acteurs. Ainsi, à Dunkerque, la centralité, la relation ville-port, la mobilité et l'identité sont apparues déterminantes dans l'élaboration du projet urbain.

Ces quatre figures sont mobilisées aujourd'hui dans la plupart des projets urbains qui concernent notamment les villes portuaires (Ile de Nantes à Nantes, Ville-port à SaintNazaire, Euroméditerranée à Marseille...). Dans l'exemple dunkerquois, les paroles d'habitants construisent la centralité uniquement dans une dimension fonctionnelle. Celle-ci parait en effet se cristalliser dans les commerces de détails qui s'agglomèrent le long de quelques rues et de quelques places. Le projet Neptune en créant notamment le centre Marine, puis le Pôle Marine a poursuivi et renforcé cette tendance en exploitant les commerces comme les éléments principaux de l'animation urbaine, soutenant ainsi le projet d'extension du centre à l'échelle d'une agglomération de deux cents mille habitants. La densité des commerces et leur diversité délimitent un espace perçu comme le centre-ville. Il s'agit d'une désincarnation de la valeur symbolique du concept même de centralité et par là peut-être du centre-ville. La question se pose alors de savoir si ce constat est lié à un projet urbain incomplet ou s'il valide la réalité de sociétés post-modernes. Le programme Euralille semble plus complet en donnant à la ville une nouvelle géométrie. Mais le projet urbain peut aussi être au cœur d'une nouvelle citadinité. En effet J. Boissonade (Hellequin dir., 2006) montre comment un projet urbain "classique » peut aussi représenter un projet de société car il s'agit de créer une citadinité spécifique à travers les conditions d'élaboration du projet.

Le projet urbain intervient alors comme un paradigme qui porte le dessein d'une ville, la forme de ses espaces mais aussi un projet de société. La confrontation des effets attendus et des effets perçus est alors extrêmement dynamique et porteur de sens. Plus largement encore que le projet, la diffusion, à toutes les échelles de territoire, du 
développement durable montre aussi l'importance des idéologies spatiales partagées tant dans les textes académiques que dans les enjeux urbains de notre siècle.

\section{Développement durable versus développement métropolitain : une synthèse possible par l'action publique}

Le « développement durable » ne peut plus aujourd'hui être ignoré des acteurs du développement des territoires. De ce point de vue, le développement urbain des grandes métropoles est critiqué en raison de ses effets dévastateurs sur l'environnement humain. Mais le développement durable renvoie également à une dimension sociale forte : préoccupation environnementale et préoccupation sociale constituent deux volets indissociables.

La critique du développement métropolitain aujourd'hui est largement justifiée, cependant que pour la première fois, dans nos métropoles, l'innovation urbaine (ex : la ville renouvelée, les plans de déplacement urbain, le HQE...) s'oriente vers des champs nouveaux, sous l'effet bénéfique du discours sur le développement durable : la problématique du renouvellement urbain occupe désormais une position centrale dans ces préoccupations.

Lille représente en France un bon exemple de mise en œuvre de stratégies de développement métropolitain. Un aspect important de cette stratégie concerne le cadre de vie et l'environnement. La gestion de l'héritage industriel, en termes sociaux et environnementaux, représente encore aujourd'hui un important défi pour ce type de territoire. Outre son importance quant à la production d'une nouvelle image, l'environnement apparaît également stratégique en tant que levier pour le développement et l'attractivité du territoire, ainsi que comme facteur d'amélioration du cadre de vie des citoyens. Le nouveau Schéma directeur mis en œuvre depuis 2003 fait ainsi de l'environnement et du cadre de vie l'une de ses priorités. Mais entre la gestion des séquelles d'un développement industriel prédateur tel que l'a connu l'Europe à partir du XIXème siècle et la mise en ouvre d'un développement durable pour le XXIème siècle, la voie est étroite, souvent difficile, entre les intérêts contradictoires des différents acteurs d'un territoire. La création de nouveaux équipements, la volonté de se donner les moyens fonciers de son développement (nouvelles zones d'activités, nouveaux secteurs résidentiels) et de créer de nouvelles infrastructures (routes, autoroutes...) sont souvent en contradiction avec les objectifs de préservation de l'environnement. Entre les principes qui font consensus et les questions qui fâchent, la question de l'environnement, à Lille comme ailleurs, fait l'objet de tensions particulières entre acteurs du développement, groupes de pressions, et mobilisations citoyennes (épisode du contournement sud de Lille, riverains de l'A27, débat sur l'A24, etc..) (Bachelet, Menerault \& Paris, 2007). L'adoption récente d'un Agenda 21 communautaire vient renforcer le champ de l'action publique en matière de protection de l'environnement et de développement métropolitain raisonné.

Traitement de friches industrielles ; schéma de trames vertes et bleues ; projet HQE ; mixité sociale et économique : le Nord Pas de Calais constitue un terrain d'expérimentation des nouvelles formes de politiques publiques régionales destinées à encourager la prise en compte du développement durable dans les stratégies urbaines.

\section{Conclusion}

Comme d'autres grandes régions urbaines de l'Europe du Nord-Ouest, profondément marquées par la période industrielle, les villes du Nord de la France sont entrées très rapidement, en moins d'un quart de siècle, dans des processus de développement que l'on peut qualifier de post-modernes : la mise en œuvre d'un développement fondé sur l'élaboration de stratégies de territoires et de projets urbains, la place croissante de la 
culture dans ces stratégies et projets, la mise en place de nouveaux modes de gouvernance urbaine, la réflexion nouvelle sur la densité à l'occasion des opérations de renouvellement urbain, l'intégration des préoccupations du développement durable, etc... illustrent cette tendance. La ville est transformée par ce processus associant dans un même lieu le passé industriel patrimonialisé au futur établi sur de nouvelles activités économiques dans lesquelles la créativité occupe une place essentielle. Elle devient alors elle-même une ville post-moderne où s'élabore un nouveau sens des lieux. Cela n'est certes pas propre aux villes de cette région, mais eu égard à la rapidité de l'évolution de ces anciennes villes industrielles, le terrain d'étude apparaît particulièrement pertinent pour illustrer la profonde mutation de la ville européenne depuis le dernier quart du XXème siècle. De la Ruhr aux villes anglaises en passant par les Pays-Bas, les références locales aux modèles proposés dans ces différents pays sont patentes. Les expérimentations menées dans ces villes du Nord - Pas de Calais et au sein du vaste système métropolitain de la région urbaine de Lille, contribuent à enrichir le débat et ont aussi valeur de modèle, à l'exemple de la notion de renouvellement urbain née dans cette région.

Ce sont ces pistes de réflexion sur la transformation de la ville qui, dans le cadre de cette nouvelle revue dédiée aux "Territoire en Mouvement », retiendront, en ce qui concerne le champ de l'urbain, l'intérêt de l'équipe de rédaction. Cet intérêt renouvelé s'inscrit logiquement dans la continuité d'« Hommes et Terres du Nord », qui, de Philippe Pinchemel à Pierre Bruyelle, en passant par Jacqueline Baujeu-Garnier ou André Gamblin, a, dès l'origine, accueilli de nombreux auteurs mobilisés par la question du devenir des villes à travers différentes approches.

\section{Bibliographie}

ASHER F., 1995, Métapolis ou l'avenir des villes. Éditions O. Jacob, Paris, 347 p.

BAUDRILLARD J., 1968, Le système des objets, la consommation des signes, Paris, Gallimard.

BACHELET F., MENERAULT Ph., Paris D., 2007 (à paraître), Logiques métropolitaines : modèles, acteurs, processus, Paris, L'Harmattan.

BONDUE J-P, 1996, Le commerce comme outil de reconquête des territoires : l'exemple du Nord - Pas-de-Calais, in Nord - Pas-de-Calais : changement régional et dynamique des territoires, FREVille, ORHA, pp. 123-138

BONDUE J-P, 2000, " L'essor de la grande distribution au miroir des processus de métropolisation et de périurbanisation dans le Nord - Pas-de-Calais », Colloque Guignet Ph. et Bondue J-P.(dir.) Les métamorphoses des réseaux urbains dans la France du Nord de Louis XIV à nos jours, Lille, Revue du Nord, $\mathrm{n}^{\circ}$ 2, pp. 537-552

DOI : 10.3406/rnord.2000.3022

BOURDIN A., 2005, La métropole des individus, l'Aube, 249 p.

BRUNET R. (ss.la dir), 1989, Les villes européennes. Datar-La Documentation française.

BRUYELLE P., 1991, La Communauté urbaine de Lille. La Documentation française, 192 p.

CLAVAL 1982, La logique des villes, Paris, Litec, 625p.

CLAVAL P. 1968, "La théorie des villes", Revue géographique de l'Est, 8, 3-56.

DOI : $10.3406 /$ rgest.1968.2008

DAMETTE F, 1994, La France en Villes. Datar - La Documentation Française, Paris, 271 p.

DESSE R-P., 2001, Le nouveau commerce urbain. Dynamiques spatiales et stratégies des acteurs, Presses universitaires de Rennes,198 p.

DOLEZ B., Paris D. (ss. la dir.), 2004, Métropoles en construction. Territoires, politiques et processus. Paris, éditions de L'Harmattan, collection Logique politique (dirigée par Y.Surel) 294 p.

DUHAMEL S., HELLEQUIN A.-P..(2005) De nouvelles échelles pour l'aire métropolitaine lilloise : recomposition des mobilités entre la métropole lilloise et le pôle urbain de DK in Colloque Logiques métropolitaines : modèles, acteurs et processus, Ifresi. Lille.

GODIER P., TAPIE, G., 2004, Recomposer la ville : mutations bordelaises. L’Harmattan, 236 p.

HAUTREUX, J., ROCHEFORT M., 1964, La fonction régionale dans l'armature urbaine française, Paris, Ministère de la Construction.

HELLEQUIN A.- P. (dir.), "Effets et réception du projet urbain dans les villes portuaires », Rapport de recherche pour le PUCA, Ministère de l'équipement, juillet 2006. 
HELLEQUIN A.- P., 2002, "Urban regime, five strategies for downtown, un essai de transposition à Dunkerque", Espace et sociétés, pp. 123-140

INGALLINA, P., 2001, Le projet urbain, Que Sais Je nº 3585, PUF, 127 p.

JALABERT G., 1995, Toulouse, métropole incomplète. Anthropos, 202 p.

JOUVE B., LEFEVRE Ch., 2002, Métropoles ingouvernables, Elsevier, 203 p.

LEMARCHAND N., "Le centre commercial, lieu de consommations et de transactions culturelles", Géographie et Culture, ${ }^{\circ}$ 53, Paris, Éditions L'Harmattan, 2005, pp. 117-122.

LEMARCHAND N., JAUMAIN S., (ss.la dir), 2007 (à paraître) La banlieue au Canada et en Europe : une perspective comparée, Peter Lang, collection études canadiennes

LUSSAULT M. 2003, « urbaine (géographie) », in Lévy J. et Lussault M. (dir.), Dictionnaire de la géographie et de l'espace des sociétés, Paris, Belin, 1034p.

LUSSAULT M., 2000, "La ville des géographes », La ville et l'urbain l'état de savoirs, Paris, Éditions de la Découverte, pp. 21-35.

MASBOUNGI A. (ss. la dir), 2002, Projets urbains en France. Éditions du Moniteur, Paris, 208 p.

METTON A., 2001, "Les temps du commerce sont-ils adaptés aux consommateurs ?" dans T. Paquot (dir.), Le quotidien urbain, essais sur les temps des villes, Paris, La Découverte / Institut des villes, p. 67-84.

MOATI P., 2001, L'avenir de la grande distribution, Paris, Odile Jacob, 392 p.

MOREL, B., 1999, Marseille, naissance d'une métropole, Paris, L’Harmattan, 222 p.

MOTTE A. (ss. la dir), 2006 (à paraitre), Les agglomérations françaises face aux défis métropolitains, Economica Anthropos, collection « villes »

Paris D. Stevens J.F., 200o, Lille et sa région urbaine : la bifurcation métropolitaine, Paris, l'Harmattan, collection Métropole 2000, 265 p.

PERON R., 1993.- La fin des vitrines- Des temples de la consommation aux usines à vendre. Éditions de l'École Normale Supérieure de Cachan, 308 p.

RATOUIS O. et SEGAUD M., 2001, "De la « maîtrise d'ouvrage » au « collectif d'énonciation » : proposition pour une nouvelle approche de la production territoriale locale", Espaces et Sociétés, 105-106, 127-145.

ROSEMBERG M., 2000, Le marketing urbain en question, Economica 188 p.

ROZENBLAT C., CICILLE P. 2003, Les villes européennes : analyse comparative. Datar-La Documentation Française, 96 p.

SALCEDO R., When the Global Meets the Local at the Mall, American Behavioral Scientist, vol. $46 \mathrm{~N}^{\circ}$ 8, April 2003 1084-1103, 2003 Sage Publications

DOI : $10.1177 / 0002764202250500$

SASSEN, S., 1991, The global City : NewYork, London, Tokyo. Princeton, Princeton University Press

STEVENS J.F., 1989, Lille Eurocité. SEP SGAR, préfecture de Région NPDC, Lille.

VALCKE S., 2003, La réparation, une approche des espaces urbains, Les Mots, 72, 29-44.

DOI : $10.4000 /$ mots.5593

VELTZ, 1997, Mondialisation, villes et territoires ; l'économie d'archipel. Paris, P.U.F., 262 p.

\section{Notes}

1 Benoît Latreille, cabinet expert Cushman \& Wakefield Healey \& Baker, LSA nº 1934 le 05-012006, site www.lsa.fr

2 Ouverture du centre en 1981, positionné comme un méga centre régional ; le WEM ajoute les activités ludiques dès 1983 qu'il complète en 1985 et en 1998.

3 LSA n ${ }^{\circ} 1700$ le 23-11-2000, www.lsa.fr

4 Valeur locative moyenne juillet 2006 : 905 Euros $/ \mathrm{m}^{2}$ (3ème rang après Paris 1085 Euros/m2 et Strasbourg 934 Euros/m2), source CB Richard Ellis, www.cbre.fr/etudes/ Étude_Commerce_2006_fr.pdf

5 Domus, inauguré en mars 2006 à Rosny-sous-Bois est le premier centre commercial de France dédié à l'équipement de la maison. Il compte 70 enseignes. Le concept de centre dédié s'est d'abord concrétisé en Europe du Nord à Amsterdam et Rotterdam.

6 J-P Bondue, Le commerce comme outil de reconquête des territoires : l'exemple du Nord - Pas de Calais , FRE-Ville 1996.

7 Établissement Public national d'Aménagement et de Restructuration des Espaces Commerciaux et Artisanaux, crée en 1996 et dirigé depuis 2006 par François Mius. 


\section{Pour citer cet article}

Référence électronique

Anne-Peggy Hellequin, Nathalie Lemarchand et Didier Paris, « Système métropolitain et logique de projet : une approche de la question urbaine en géographie et aménagement », Territoire en mouvement Revue de géographie et aménagement [En ligne], 1 | 2006, mis en ligne le 01 septembre 2010, consulté le 15 septembre 2020. URL : http://accesdistant.bu.univparis8.fr:2179/tem/120 ; DOI : https://accesdistant.bu.univ-paris8.fr:2118/10.4000/tem.120

\section{Cet article est cité par}

- Ayerbe, Delphine. (2015) La place de l'eucalyptus à Addis-Abeba. Territoire en mouvement. DOI: 10.4000/tem.3132

\section{Auteurs}

Anne-Peggy Hellequin

Université du Littoral Côte d'Opale - Institut des Mers du Nord

24 Quai de la Citadelle

59140 Dunkerque

Articles du même auteur

Faire ou non confiance aux gestionnaires du risque ? [Texte intégral]

Le cas d'un village entouré de sites Seveso

Paru dans Territoire en mouvement Revue de géographie et aménagement, 1| 2007

Compte-rendu de la conférence débat de juin 2007 sur l'état et l'avenir des revues universitaires de géographie en langue française [Texte intégral]

Paru dans Territoire en mouvement Revue de géographie et aménagement, 3 | 2007

Nathalie Lemarchand

Université de Valenciennes

Le Mont Houy

59313 Valenciennes CEDEX 9

Articles du même auteur

Deboudt P., Le Blanc A., Lemarchand N., Wayens B. (coord.), 2018, Forum des revues francophones de géographie et ouvertes à la géographie / aux géographes. État des lieux, enjeux et coopérations futures [Texte intégral]

Jeudi 22 novembre 2018, MESHS, Lille

Paru dans Territoire en mouvement Revue de géographie et aménagement, 41 | 2019

Corentin Sellin, Amérique du Nord, entre intégration et fragmentation [Texte intégral]

2014, Ellipses : Collection 50 fiches, 120 pages

Paru dans Territoire en mouvement Revue de géographie et aménagement, 30 | 2016

Jean-Claude Marsan : Montréal et son aménagement. Vivre la ville [Texte intégral]

2012, Presses de l'université du Québec, coll. Patrimoine urbain, 304 pages

Paru dans Territoire en mouvement Revue de géographie et aménagement, 23-24 | 2014

Jean-Marc Zaninetti : Les espaces de l'Amérique du Nord ; Canada, Etats-Unis, Mexique [Texte intégral]

2012, PUF : collection MAJOR, 212 pages

Paru dans Territoire en mouvement Revue de géographie et aménagement, 23-24 | 2014

Hervé Vieillard-Baron : Banlieues et périphéries. Des singularités françaises aux réalités mondiales [Texte intégral]

2ème édition revue et augmentée, Paris, Hachette supérieur, Carré Géographie, 304 pages

Paru dans Territoire en mouvement Revue de géographie et aménagement, 17-18 | 2013

Pierre Merlin : Les Grands Ensembles. Des discours utopiques aux « quartiers

sensibles » [Texte intégral]

La Documentation française, 2010, 209 pages

Paru dans Territoire en mouvement Revue de géographie et aménagement, 13 | 2012

Tous les textes...

Didier Paris

Université Lille 1

Laboratoire TVES EA 4019 
UFR de Géographie et Aménagement

Avenue Paul Langevin

59655 Villeneuve d'Ascq CEDEX

Articles du même auteur

Éditorial [Texte intégral]

Paru dans Territoire en mouvement Revue de géographie et aménagement, 2 | 2006

\section{Droits d'auteur}

(c) (†)

Territoire en mouvement est mis à disposition selon les termes de la licence Creative Commons Attribution 4.0 International. 